\title{
Nondestructive Optical Measurement of Refractive-index Profile of Graded-index Lenses
}

\author{
Byounghwak Lee, Naeho Shin, Kwan Jeong, Myoung-Jin Park, and Byung-Gyu Kim \\ Department of Physics and Chemistry, Korea Military Academy, Seoul 139-799, Korea \\ Jang-Hoon Yoo \\ DMC RछD Center, Samsung Electronics Co., Ltd., Suwon 443-742, Korea \\ Dae-Geun Kim, Ki-Hyuck Yun, Kewseung Lee, Kyung-Hwan Kim, Dae-Kyu Kim, \\ and Seung-Han Park* \\ Institute of Physics and Applied Physics, Yonsei University, Seoul 120-749, Korea
}

(Received May 25, 2009 : revised September 25, 2009 : accepted September 25, 2009)

\begin{abstract}
We propose a simple nondestructive method to obtain refractive-index profiles of a graded-index (GRIN) light-focusing rod by means of a diffraction grating. In our proposed method, a laser beam is illuminated through a diffraction grating perpendicular to the axis of the GRIN lens and the separation between the zeroth and first-order diffraction peaks is measured and analyzed. The results demonstrate that the refractive-index profiles of commercially available GRIN lenses can be successfully reconstructed.
\end{abstract}

Keywords: Refractive-index profile, Graded-index lens, Diffraction, Grating

OCIS codes : (110.2760) Gradient-index lenses; (290.3030) Index measurements; (050.1950) Diffraction gratings; (060.2300) Fiber measurements

\section{INTRODUCTION}

In the last decades, gradient-index (GRIN) materials have been widely utilized in science and technology. In particular, many different types of GRIN rod lenses have been developed for on-axis and off-axis imaging, focusing, and collimation in optical systems $[1,2]$. Linear arrays of GRIN lenses have been employed in laser printers, photocopiers, and fax machines [3-6]. Planar GRIN waveguides such as directional couplers and wavelength-division multiplexers have been realized for the integrated optical circuitry [7, 8]. GRIN plastic optical fibers (GI-POFs) have also attracted much attention for short distance networks due to their high bandwidth transmission capabilities [9-11].

The quality and efficiency of GRIN materials are heavily dependent upon the shape of the radial refractive-index profile. For instance, the bandwidth of a large-core GI-POF can be maximized when the distribution of the refractive-index in the radial direction of the fiber is parabolic [12]. A quadratic index profile of a GRIN lens can minimize the monochromatic aber- rations. Therefore, various optical techniques to measure the refractive index profiles of GRIN structures have been extensively investigated, including beam deflection technique [13, 14], prism technique [15], longitudinal and transverse interferometry [16-19], and shearing interferometry [20, 21]. However, these conventional techniques require either complicated experimental arrangements or precise polishing processes.

In this paper, a simple nondestructive method to acquire refractive-index profiles of GRIN light-focusing rods (i.e., GRIN lens) is proposed. The method involves illuminating a laser beam through a diffraction grating perpendicular to the axis of a GRIN lens and recording the diffraction patterns at the focal plane of the GRIN lens. In particular, the separation between the zeroth and first-order diffraction patterns is measured and analyzed. The results demonstrate that the radial distribution of refractive-index of a commercially available GRIN lens can be successfully reconstructed by our proposed method.

\footnotetext{
*Corresponding author: shpark@yonsei.ac.kr
} 


\section{EXPERIMENTAL SETUP AND REFRACTIVE-INDEX DETERMINATION}

The refractive-index of GRIN lenses varies continuously with radial distance from the axis. In general, the radial distribution of the refractive index of a GRIN lens can be written as [22]

$$
n(r)=n_{c}\left(1-\frac{A^{2}}{2} r^{2}\right)
$$

where $n_{c}$ is the refractive-index at the axis of GRIN lens, $A$ is the gradient parameter determining index profile, and $r$ is the radial distance from the lens axis.

Fig. 1 shows a schematic diagram of our experimental setup to obtain the refractive-index profile of GRIN lens. He-Ne laser and a chrome-coated Ronchi gratings are used for producing plane waves and diffraction patterns, respectively. If a laser beam is illuminated through a diffraction grating perpendicular to the axis of a GRIN lens, as displayed in Fig. 1, the Fraunhofer diffraction patterns can be observed at the focal plane. The distance between the zeroth-order principal maximum and the first order diffraction peak in the paraxial region is given by [23]

$$
d=f \frac{\lambda}{p}
$$

where $f$ is an effective focal length of the GRIN lens, $\lambda$ is the wavelength of the incident beam, and $p$ is the pitch size of the grating, respectively. Note that the wavefront suffers bending of its path as a result of differential phase delays due to the varying refractiveindex of the GRIN lens, containing information to reconstruct the index profile.

In order to get the index profile from the experimental data, at first, we assume that GRIN lens has a constant refractive-index $n_{o}$ for $r \leq R$ and $n_{o}$ is the refractive-index of the outermost part of the GRIN lens, as shown in Fig. 2(a). If the GRIN lens is located inside index-matching oil of $n_{\text {oil }}$, its focal length is given by $[24]$

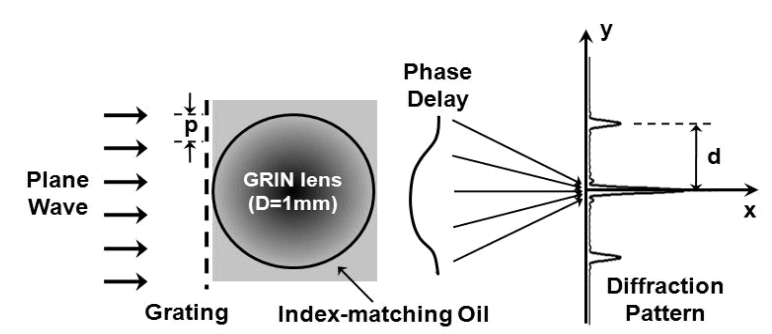

FIG. 1. Schematic diagram of experimental setup for measuring refractive-index profile of GRIN lenses.

$$
\frac{n_{o}}{f-R}=\left(n_{o}-n_{\text {oil }}\right) \frac{2}{R}
$$

where $f$ is a focal length, $R$ is the radius of the GRIN lens, and $n_{\text {oil }}$ is the refractive-index of index-matching oil.

However, in our experiment, the GRIN lens is located partially in the index-matching oil as shown in Fig. 2(b). The incident ray impinged on the GRIN lens is deflected and produces the internal angle $\alpha$ and the intersection angle $\theta_{f}$. If the minimum distance from the center of the GRIN lens to the ray is denoted by $r^{*}$, the focal length can be approximately written by [25]

$$
f \cong \frac{y}{n_{\text {oil }}(2 y / R+\alpha-\pi)} \quad \text {, where } \quad \alpha=2 \int_{r^{*}}^{R} \frac{n_{\text {oil }} y}{r \sqrt{[n(r) r]^{2}-\left(n_{\text {oil }} y\right)^{2}}} d r
$$

The $r^{*}$ is the real root of the following equation,

$$
n_{c} A^{2} r^{3}-2 n_{c} r+2 n_{\text {oil }} y=0 \quad\left(0<r^{*} \leq R\right)
$$

which makes the denominator in the integral to be equal to zero.

As a second step, we assume that the index of refraction of the GRIN lens drops off parabolically from the central axis (shown in Fig. 2(b)), as described in Eq. (1), and the refractive-index of the outermost part of GRIN lens is given by $n_{0}$. The GRIN lens used in our experiment is of $1.0 \mathrm{~mm}$ in diameter and $n_{c}$ can be expressed as

$$
n_{c}=\frac{2 n_{o}}{2-A^{2} R^{2}}
$$

by substituting $r=R$ into Eq. (1). Therefore, the refractive-index profile of the GRIN lens can be reconstructed by measuring the distance $d$ to get $f$ from Eq. (2) and finding $\alpha$ from Eq. (4) with given parameters like $R, n_{\text {oil }}$ and $y$. Finally, the gradient parameter $A$ is obtained by solving the integral equation in Eq. (4).

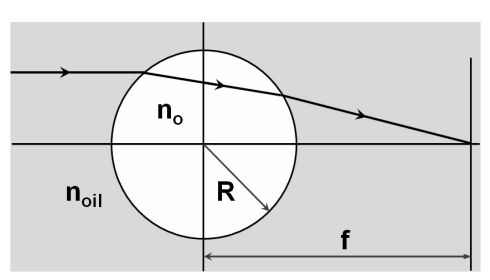

(a)

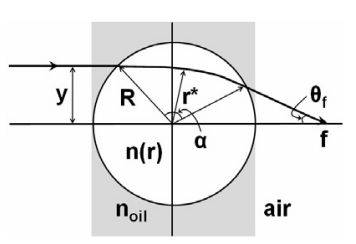

(b)
FIG. 2. The focal length of the GRIN lenses with (a) a constant refractive-index and (b) a graded refractiveindex. 


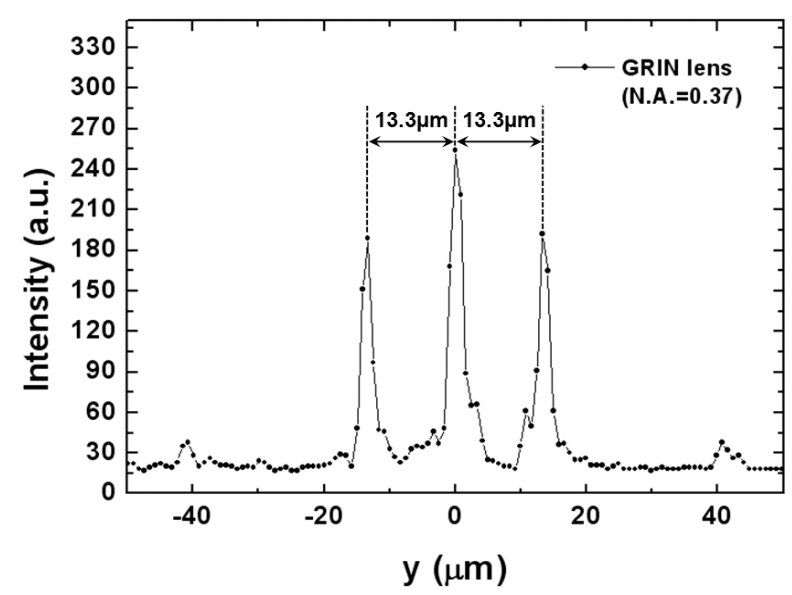

FIG. 3. Diffraction pattern of a GRIN lens with N.A. of 0.37 .

Fig. 3 shows a typical diffraction pattern observed by a charge-coupled-device (CCD) camera for a commercial GRIN lens with numerical aperture (N.A.) of 0.37 at the focal plane. In order to measure the separation between the $0^{\text {th }}$-order and $1^{\text {st }}$-order diffraction peaks accurately, both diffraction grating and GRIN lens were simultaneously moved by a pico-motor actuator with less than $100 \mathrm{~nm}$ resolution. By carefully monitoring the position of the 0th-order principal peak in the CCD camera as a function of moving distance by the pico-motor, we could confirm that the measurement accuracy of the distance between the 0th-order and 1st-order peaks is less than 0.5 $\mu \mathrm{m}$. We would like to mention that the effective focal distance of the GRIN lens can be obtained from this measured separation between the $0^{\text {th }}$ and $1^{\text {st }}$-order peaks by using the Eq. (2).

\section{RESULTS AND DISCUSSIONS}

The measured separation $(d)$ between the $0^{\text {th }}$-order and the $1^{\text {st }}$-order diffraction peaks is found to be $13.3 \pm 0.1 \mu \mathrm{m}$ for a SELFOC ${ }^{\circledR}$ GRIN lens of N.A. $=0.37$. From Eq. (2), therefore, the effective focal length $(f)$ is obtained to be $1.7865 \mathrm{~mm}$ with the parameters of $d=13.3 \mu \mathrm{m}, \lambda=0.6328 \mu \mathrm{m}$, and $p=85.0 \mu \mathrm{m}$. From Eq. (6), $n_{c}$ can be expressed by $A$ with a given index of refraction $\left(n_{0}\right)$ of 1.5153 and $R=0.5 \mathrm{~mm}$. Then, by assuming paraxial approximation $(y \leq R / 10)$ and refractive index of matching oil, $n_{\text {oil }}$, of $1.4584, r^{*}$ is obtained as a function of $A$. Finally, by inserting this $r^{*}$ in the Eq. (4), the gradient parameter determining index profile, $A$, is determined to be 0.5025 , which is in good agreement with the parameter given by the manufacturer [26].

Fig. 4 shows the reconstructed refractive-index profiles obtained by our proposed method and the index profiles given by the company $\left(\mathrm{SELFOC}^{\mathrm{R}}\right)$ for

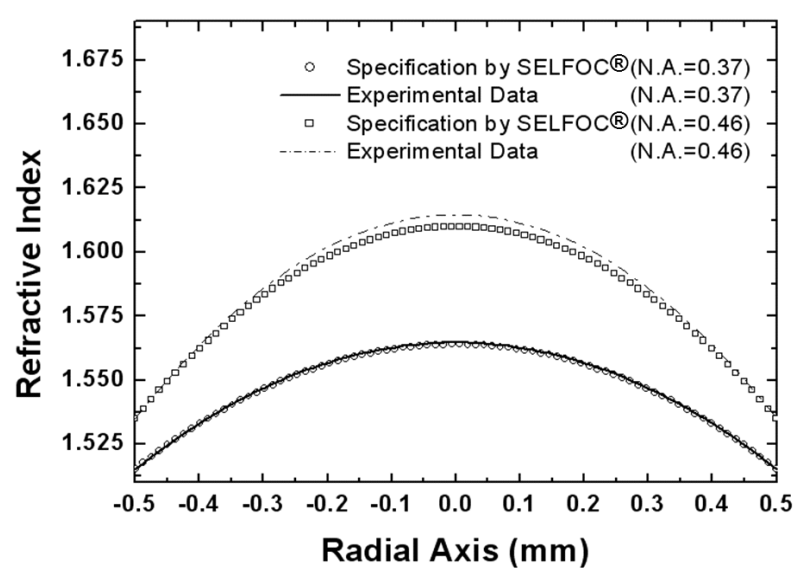

FIG. 4. The reconstructed refractive-index of a GRIN lens and its technical data given by the SELFOC ${ }^{\mathbb{R}}$.

TABLE 1. A summary of various factors determining refractive-index profile of GRIN lenses

\begin{tabular}{l|c|c}
\hline \hline & $\begin{array}{c}\text { GRIN Lens } \\
\text { (N.A.=0.37) }\end{array}$ & $\begin{array}{c}\text { GRIN Lens } \\
\text { (N.A. }=0.46)\end{array}$ \\
\hline $\begin{array}{l}\text { Outermost } \\
\text { Refractive index } n_{o}\end{array}$ & 1.5153 & 1.5351 \\
\hline $\begin{array}{l}\text { Position of } 1^{\text {st }} \text { order } \\
\text { diffraction pattern } d(\mu \mathrm{m})\end{array}$ & 13.3 & 9.1 \\
\hline $\begin{array}{l}\text { Effective focal } \\
\text { length } f(\mathrm{~mm})\end{array}$ & 1.7865 & 1.2223 \\
\hline $\begin{array}{l}\text { Minimum } \\
\text { distance } r^{*}(\mu \mathrm{m})\end{array}$ & $\begin{array}{l}0.5025 \\
(0.499)\end{array}$ & $\begin{array}{c}0.6278 \\
(0.610)\end{array}$ \\
\hline $\begin{array}{l}\text { Profile shape } \\
\text { parameter } A\end{array}$ & $\begin{array}{l}1.5647 \\
(1.564)\end{array}$ & $\begin{array}{c}1.6146 \\
(1.610)\end{array}$ \\
\hline $\begin{array}{l}\text { Refractive index } \\
\text { of center } n_{c}\end{array}$ & $\begin{array}{c}0.0007 \\
\text { or } 0.04 \%\end{array}$ & $\begin{array}{c}0.0046 \\
\text { or } 0.29 \%\end{array}$ \\
\hline $\begin{array}{l}\text { Error in refractive } \\
\text { index of center }\end{array}$ &
\end{tabular}

GRIN lenses of N.A. $=0.37$ and N.A. $=0.46$. The result reveals that errors in refractive indices of $n_{c}$ 's are 0.0007 and 0.0046 , indicating only $0.04 \%$ and $0.29 \%$ deviations from the $n_{c}$ values provided by the company. All the acquired parameters are summarized in Table 1. The values of $n_{c}$ and $A$ in parentheses in the Table 1 are the specification data from the company. The error of a GRIN lens with N.A. $=0.46$ has larger than that of a GRIN lens with N.A. $=0.37$. Considering our paraxial approximation, it can be expected that the errors in refractive-index and index profile can be increased with increasing N.A. Measurements of refractive index profiles of various GRIN lenses with different N.A.'s are in progress to find the theoretical and experimental limits of our approximation and proposed method. 


\section{CONCLUSIONS}

A simple nondestructive method to measure the refractive-index profile of GRIN focusing rods has been demonstrated by means of a diffraction grating. In particular, the separation between the zeroth and the first-order diffraction peaks are utilized to obtain the index difference in the center and the outermost region of the GRIN lens as well as its index profile. The results show that the refractive-index profiles of commercially available GRIN lenses can be successfully reconstructed. In addition, we show that the refractive indices at the center of GRIN lenses have only $0.04 \%$ and $0.29 \%$ discrepancies, comparing with the index values provided by the manufacturer. We believe that our proposed method can be useful to get the refractive-index profiles of GRIN materials nondestructively and to control the quality of GRIN elements in the manufacturing process.

\section{ACKNOWLEDGMENT}

This research was supported by Korea Military Academy, the Next Generation Emerging Technology Program of the Ministry of Knowledge Economy, Brain Korea 21(BK21) Project and Pioneer Research Center program (Contract No. 2009-008-1527) through the National Research Foundation of Korea funded by the Ministry of Education, Science \& Technology.

\section{REFERENCES}

1. C. G. Reino, M. V. Perez, and C. Bao, Gradient-index Optics (Springer, Berlin, Germany, 2002).

2. D. T. Moore, "Gradient-index optics: a review," Appl. Opt. 19, 1035-1038 (1980).

3. Y. Koike, Y. Takezawa, and Y. Ohtsuka, "New interfacialgel copolymerization technique for steric GRIN polymer optical waveguides and lens arrays," Appl. Opt. 27, 486-491 (1988).

4. C. Ye and R. R. McLeod, "GRIN lens and lens array fabrication with diffusion-driven photopolymer," Opt. Lett. 33, 2575-2577 (2008).

5. H. Kobayashi and T. Horiuchi, "Novel projection exposure system using gradient-index lens array," Jpn. J. Appl. Phys. 47, 5702-5707 (2008).

6. F. Okano, M. Kobayashi, J. Arai, and M. Okui, "Depth control GRIN lens array for integral photography," Proc. SPIE 5243, 30-41 (2003).

7. S. Janz, B. Lamontagne, A. Delage, A. Bogdanov, D. $\mathrm{X}$. Xu, and K. P. Yap, "Single layer a-Si GRIN waveguide coupler with lithographically defined facets," in Proc. 2nd IEEE International Conference on Group IV Photonics (Antwerp, Belgium, Sep. 2005), pp. 129-131.

8. S. Rios, R. Srivastava, and C. G. Reino, "Coupling of single-mode fibers to single-mode GRIN waveguides by butt-joining," Opt. Comm. 119, 517-522 (1995).
9. S. C. J. Lee, F. Breyer, S. Randel, B. Spinnler, I. L. L. Polo, D. van den Borne, J. Zeng, H. P. A. van den Boom, and A. M. J. Koonen, "Performance of maximum likelihood sequence estimation in $10 \mathrm{~Gb} / \mathrm{s}$ transmission systems with polymer optical fiber," in Proc. Symposium IEEE/LEOS Benelux Chapter (Eindhoven, Netherlands, Nov. 2006), pp. 17-19.

10. I. T. Monroy, H. P. A. vd Boom, A. M. J. Koonen, G. D. Khoe, Y. Watanabe, Y. Koike, and T. Ishigure, "Data transmission over polymer optical fibers," Optical Fiber Technology 9, 159-171 (2003).

11. D. G. Kim, S. Y. Woo, D. K. Kim, T. Y. Hwang, D. Y. Kim, and S.-H. Park, "Generation of 1.5 Gbps pseudo-random binary sequence optical signals by using a gain switched Fabry-Perot semiconductor laser," J. Opt. Soc. Korea 9, 103-106 (2005).

12. W. C. Chen, Y. Chang, and J. P. Hsu, "Theoretical analysis on a multilayer coextrusion process for preparing gradient-index polymer optical fibers," J. Phys. Chem. B 103, 7584-7590 (1999).

13. P. L. Chu, "Nondestructive measurement of index profile of an optical-fibre preform," Electron. Lett. 13, 736-738 (1977).

14. L. S. Watkins, "Laser beam refraction transversely through a graded-index preform to determine refractive-index ratio and gradient profile," Appl. Opt. 18, 2214-2222 (1979).

15. H. H. Lim, M. S. Kwon, H. J. Choi, B. J. Kim, and M. Cha, "Measurement of refractive index of solid medium by critical angle method when air gap is present," J. Opt. Soc. Korea 12, 210-214 (2008).

16. W. E. Martin, "Refractive index profile measurements of diffused optical waveguides," Appl. Opt. 13, 2112-2116 (1974).

17. J. Stone and R. M. Derosier, "Elimination of errors due to sample polishing in refractive index profile measurements by interferometry," Rev. Sci. Instru. 47, 885-887 (1976).

18. M. E. Marhic, P. S. Ho, and M. Epstein, "Nondestructive refractive-index profile measurements of clad optical fibers," Appl. Phys. Lett. 26, 574-575 (1975).

19. M. J. Saunders and W. B. Gardner, "Nondestructive interferometric measurement of the delta and alpha of clad optical fibers," Appl. Opt. 16, 2368-2371 (1977).

20. Y. Ohtsuka and Y. Shimizu, "Radial distribution of the refractive index in light-focusing rods: determination using interphako interference microscopy," Appl. Opt. 16, 1050-1053 (1979).

21. Y. Kokubun and K. Iga, "Refractive-index profile measurement of preform rods by a transverse differential interferograms," Appl. Opt. 19, 846-851 (1980).

22. K. S. R. Krishna and A. Sharma, "Chromatic aberrations of radial gradient-index lenses. II. Selfoc lenses," Appl. Opt. 35, 1037-1040 (1996).

23. J. W. Goodman, Introduction to Fourier Optics (McGrawHill Book Co., Singapore, 1996), Chapter 4.

24. F. G. Smith, T. A. King, and D. Wilkins, Optics and Photonics (John Wiley \& Sons, Ltd., West Sussex, UK, 2007), Chapter 2.

25. S. P. Morgan, "General solution of the Luneberg lens problem,” J. Appl. Phys. 29, 1358-1368 (1958).

26. Gradient-index lenses, "Gradient-index lenses, antireflection coated, for $\lambda=632.8 \mathrm{~nm}$," (Product number, 06 LGD 111), http://www.mellesgriot.com/pdf/0015.16-15.20.pdf. 